\title{
Kinetics and mechanism of oxidation of aliphatic primary alcohols by quinolinium bromochromate
}

\author{
SONU SARASWAT, VINITA SHARMA and K K BANERJI* \\ Department of Chemistry, JNV University, Jodhpur 342 005, India \\ e-mail: banerjikk@rediffmail.com
}

MS received 4 December 2001; revised 2 November 2002

\begin{abstract}
Oxidation of nine aliphatic primary alcohols by quinolinium bromochromate $(\mathrm{QBC})$ in dimethylsulphoxide leads to the formation of the corresponding aldehydes. The reaction is first order with respect to both QBC and the alcohol. The reaction is catalysed by hydrogen ions. The hydrogen-ion dependence has the form: $k_{\mathrm{obs}}=a+b\left[\mathrm{H}^{+}\right]$. The oxidation of $\left[1,1-{ }^{2} \mathrm{H}_{2}\right]$ ethanol $\left(\mathrm{MeCD}_{2} \mathrm{OH}\right)$ exhibits a substantial primary kinetic isotope effect. The reaction has been studied in nineteen different organic solvents. The solvent effect was analysed using Taft's and Swain's multiparametric equations. The rate of oxidation is susceptible to both polar and steric effects of the substituents. A suitable mechanism has been proposed.
\end{abstract}

Keywords. Aliphatic alcohols; hydride ion transfer; quinolinium bromochromate.

\section{Introduction}

Quinolinium bromochromate (QBC) has been used as a mild and selective oxidizing reagent in synthetic organic chemistry. ${ }^{1}$ There seem to be no reports on the kinetic and mechanistic aspects of oxidation reactions by QBC. The kinetics of oxidation of alcohols by QBC has also not been reported. We have been interested in the kinetics of reactions of complexed $\mathrm{Cr}(\mathrm{VI})$ species and have already reported such studies of the oxidation of primary aliphatic alcohols by other pyridinium and quinolinium halochromates. ${ }^{2-4}$ In this paper we describe the kinetics and mechanism of the oxidation of nine primary aliphatic alcohols by QBC in dimethylsulphoxide (DMSO) as solvent.

\section{Experimental}

\subsection{Materials}

QBC was prepared by the reported method $^{1}$ and its purity was checked by iodometry. Procedures used for the purification of the alcohols have been described earlier. ${ }^{5}[1,1$ ${ }^{2} \mathrm{H}_{2}$ ]Ethanol $\left(\mathrm{MeCD}_{2} \mathrm{OH}\right)$ was prepared by Kalpan's method. ${ }^{6}$ Its isotopic purity, as ascertained by its NMR spectra, was $93 \pm 2 \%$. Due to the non-aqueous nature of the medium, $p$-toluenesulphonic acid ( $\mathrm{TsOH}$ ) was used as a source of hydrogen ions. TsOH is a strong acid and in a polar medium like DMSO is likely to be completely ionised. Solvents were purified by the usual methods. ${ }^{7}$

\footnotetext{
*For correspondence
} 


\subsection{Product analysis}

Product analysis was carried out under kinetic conditions. In a typical experiment, ethanol $(2.30 \mathrm{~g}, 0.05 \mathrm{~mol})$ and QBC (3.10 g, 0.01 mol) were made up to $50 \mathrm{~cm}^{3}$ in DMSO and kept in the dark for about $15 \mathrm{~h}$ to ensure completion of the reaction. The solution was then treated with an excess $\left(200 \mathrm{~cm}^{3}\right)$ of a saturated solution of 2,4-dinitrophenylhydrazine in $2 \mathrm{~mol} \mathrm{dm}{ }^{-3} \mathrm{HCl}$ and kept overnight in a refrigerator. The precipitated 2,4-dinitrophenylhydrazone (DNP) was filtered off, dried, weighed, recrystallized from ethanol and weighed again. The yield of DNP before and after recrystallization was $2.0 \mathrm{~g}$ (90\%) and $1.7 \mathrm{~g}(75 \%)$, respectively. This DNP was found identical (m.p. and mixed m.p.) to the DNP of acetaldehyde. Similar experiments with other alcohols led to the formation of the DNP of the corresponding carbonyl compounds in yields ranging from 71 to $83 \%$, after recrystallization. Iodometric determinations of the oxidation state of chromium in completely reduced reaction mixtures indicated that the oxidation state of the reduced chromium species was $3 \cdot 90 \pm 0 \cdot 15$.

\subsection{Kinetic measurements}

The reactions were followed under pseudo-first-order conditions by keeping a large excess $(\times 15$ or greater $)$ of the alcohol over QBC. The temperature was kept constant to $\pm 0 \cdot 1 \mathrm{~K}$. The solvent was DMSO, unless specified otherwise. The reactions were followed by monitoring the decrease in the concentration of QBC spectrophotometrically at $356 \mathrm{~nm}$ for $80 \%$ of the reaction. The pseudo-first-order rate constants, $k_{\text {obs }}$, were evaluated from the linear $(r=0.990-0.999)$ plots of $\log$ [QBC] against time. Duplicate kinetic runs showed that the rate constants were reproducible to within $\pm 3 \%$. The second order rate constant, $k_{2}$, was evaluated from the relation $k_{2}=k_{\text {obs }} /[$ alcohol]. Simple and multivariate linear regression analyses were carried out by the least-squares method on a personal computer.

\section{Results}

The oxidation of alcohols results in the formation of corresponding aldehydes. The overall reaction may be represented,

$$
\mathrm{RCH}_{2} \mathrm{OH}+\mathrm{CrO}_{2} \mathrm{BrO}^{-} \mathrm{QH}^{+} \rightarrow \mathrm{RCHO}+(\mathrm{HO})_{2} \mathrm{CrBrO}^{-} \mathrm{QH}^{+}
$$

Thus QBC undergoes a two-electron change. This accords with the earlier observations with pyridinium fluoro- and chloro-chromates. ${ }^{8,9}$

\subsection{Rate law}

The reaction is first order with respect to QBC. Further the pseudo-first-order rate constant, $k_{\mathrm{obs}}$, is independent of the initial concentration of QBC. The reaction is firstorder with respect to the alcohol also.

\subsection{Induced polymerization of acrylonitrile}

Oxidation of alcohols, in an atmosphere of nitrogen, failed to induce polymerisation of acrylonitrile. Further, the addition of acrylonitrile did not affect the rate. This indicates that one-electron oxidation, giving rise to free radicals, is unlikely in the present reaction. 


\subsection{Effect of temperature}

The rates of oxidation of ten primary alcohols were determined at different temperatures and the activation parameters were calculated (table 1).

\subsection{Kinetic isotope effect}

To ascertain the importance of cleavage of the $\alpha-\mathrm{C}-\mathrm{H}$ bond in the rate-determining step, oxidation of $\left[1,1-{ }^{2} \mathrm{H}_{2}\right]$ ethanol was studied. The results showed the presence of a substantial primary kinetic isotope effect (table 1).

\subsection{Effect of acidity}

The reaction is catalysed by hydrogen ions (table 2 ). The hydrogen-ion dependence has the following form below, (2). The values of $a$ and $b$, for ethanol, are 1.70 $\pm 0.02 \times$ $10^{-3} \mathrm{~s}^{-1}$ and $1.71 \pm 0.03 \times 10^{-3} \mathrm{~mol}^{-1} \mathrm{dm}^{3} \mathrm{~s}^{-1}$ respectively $\left(r^{2}=0.9985\right)$.

$$
k_{\mathrm{obs}}=a+b\left[\mathrm{H}^{+}\right] \text {. }
$$

\subsection{Solvent effect}

The oxidation of ethanol was studied in 19 different organic solvents. The choice of solvent was limited due to the solubility of QBC and its reaction with primary and secondary alcohols. There was no reaction with the solvents chosen. The kinetics were similar in all the solvents. The values of $k_{2}$ are recorded in table 3.

Table 1. Rate constants and activation parameters for oxidation of alcohols, $\mathrm{RCH}_{2} \mathrm{OH}$, by QBC

\begin{tabular}{|c|c|c|c|c|c|c|c|}
\hline \multirow{2}{*}{$\begin{array}{l}\text { Alcohol } \\
\text { (R) }\end{array}$} & \multicolumn{4}{|c|}{$10^{5} k_{2}\left(\mathrm{dm}^{3} \mathrm{~mol}^{-1} \mathrm{~s}^{-1}\right)$ at temp. $(\mathrm{K})$} & \multirow{2}{*}{$\begin{array}{c}\Delta H^{\#} \\
\left(\mathrm{~kJ} \mathrm{~mol}^{-1}\right)\end{array}$} & \multirow{2}{*}{$\begin{array}{c}\Delta S^{\#} \\
\left(\mathrm{~J} \mathrm{~mol}^{-1} \mathrm{~K}^{-1}\right)\end{array}$} & \multirow{2}{*}{$\begin{array}{c}\Delta G^{\#} \\
\left(\mathrm{~kJ} \mathrm{~mol}{ }^{-1}\right)\end{array}$} \\
\hline & 288 & 298 & 308 & 318 & & & \\
\hline $\mathrm{H}$ & 1.57 & $5 \cdot 25$ & $15 \cdot 5$ & $42 \cdot 7$ & $81 \cdot 2 \pm 0.4$ & $-55 \pm 1$ & $97 \cdot 5 \pm 0 \cdot 3$ \\
\hline $\mathrm{Me}$ & $72 \cdot 6$ & 168 & 355 & 762 & $56 \cdot 9 \pm 0 \cdot 4$ & $-108 \pm 1$ & $88 \cdot 9 \pm 0 \cdot 3$ \\
\hline Et & 118 & 263 & 536 & 1100 & $54.5 \pm 0.4$ & $-112 \pm 2$ & $88.8 \pm 0.4$ \\
\hline$n$-Pr & 198 & 415 & 770 & 1590 & $49.7 \pm 0.9$ & $-124 \pm 3$ & $86 \cdot 6 \pm 0 \cdot 8$ \\
\hline$n-\mathrm{Bu}$ & 222 & 460 & 880 & 1730 & $49 \cdot 3 \pm 0 \cdot 5$ & $-125 \pm 2$ & $86 \cdot 4 \pm 0.4$ \\
\hline$i$-Pr & 321 & 627 & 1160 & 2280 & $46.9 \pm 0.8$ & $-130 \pm 3$ & $85 \cdot 6 \pm 0 \cdot 7$ \\
\hline $\mathrm{ClCH}_{2}$ & $2 \cdot 19$ & 6.18 & $15 \cdot 6$ & 39.5 & $70 \cdot 6 \pm 0 \cdot 4$ & $-89 \pm 1$ & $97 \cdot 1 \pm 0 \cdot 3$ \\
\hline $\mathrm{MeOCH}_{2}$ & $14 \cdot 0$ & $37 \cdot 1$ & $81 \cdot 2$ & 185 & $62 \cdot 4 \pm 0.9$ & $-102 \pm 3$ & $92.7 \pm 0.7$ \\
\hline$t-\mathrm{Bu}$ & 2720 & 4440 & 6500 & 10500 & $31.2 \pm 0.9$ & $-167 \pm 3$ & $80 \cdot 8 \pm 0 \cdot 7$ \\
\hline $\mathrm{MeCD}_{2}$ & $20 \cdot 9$ & $44 \cdot 8$ & $92 \cdot 3$ & 197 & $59 \cdot 9 \pm 0.6$ & $-112 \pm 2$ & $93 \cdot 2 \pm 0.5$ \\
\hline$k_{\mathrm{H}} / k_{\mathrm{D}}$ & $5 \cdot 85$ & $5 \cdot 68$ & $5 \cdot 46$ & $5 \cdot 18$ & & & \\
\hline
\end{tabular}

Table 2. Dependence of the reaction rate on hydrogen-ion concentration.

[Alcohol] 0.10 mol dm${ }^{-3}$; [QBC] 0.001 mol dm${ }^{-3}$; Temp. $298 \mathrm{~K}$

\begin{tabular}{lcccccc}
\hline$[\mathrm{TsOH}] / \mathrm{mol} \mathrm{dm}^{-3}$ & $0 \cdot 10$ & $0 \cdot 20$ & $0 \cdot 40$ & $0 \cdot 60$ & $0 \cdot 80$ & $1 \cdot 00$ \\
$10^{4} k_{\mathrm{obs}} / \mathrm{s}^{-1}$ & $18 \cdot 5$ & $20 \cdot 6$ & $24 \cdot 1$ & $27 \cdot 0$ & $30 \cdot 9$ & $34 \cdot 0$ \\
\hline
\end{tabular}


Table 3. Effect of solvents on the oxidation of 2-propanol by BPCC at $288 \mathrm{~K}$.

\begin{tabular}{lclc}
\hline Solvents & $10^{5} k_{2}\left(\mathrm{~s}^{-1}\right)$ & \multicolumn{1}{c}{ Solvents $\left(\mathrm{s}^{-1}\right)$} & $10^{5} k_{2}$ \\
\hline Chloroform & $44 \cdot 7$ & Acetic acid & $7 \cdot 24$ \\
1,2-Dichloroethane & $55 \cdot 0$ & Cyclohexane & $1 \cdot 29$ \\
Dichloromethane & $47 \cdot 9$ & Toluene & $12 \cdot 3$ \\
DMSO & 168 & Acetophenone & $81 \cdot 3$ \\
Acetone & $43 \cdot 7$ & THF & $20 \cdot 0$ \\
N,N-Dimethylformamide & $89 \cdot 1$ & $t$-Butyl alcohol & $17 \cdot 8$ \\
Butanone & $30 \cdot 2$ & 1,4-Dioxane & $22 \cdot 9$ \\
Nitrobenzene & $57 \cdot 5$ & 1,2-Dimethoxyethane & $11 \cdot 0$ \\
Benzene & $14 \cdot 8$ & Carbon disulphide & $5 \cdot 13$ \\
Ethyl acetate & $16 \cdot 2$ & & \\
\hline
\end{tabular}

\section{Discussion}

The activation enthapies and entropies of the oxidation of the nine aliphatic alcohols are linearly related $(r=0.9925)$. The isokinetic temperature is $467 \pm 22 \mathrm{~K}$. The correlation was tested and found genuine by applying Exner's criterion. ${ }^{10}$ Exner's plot between $\log k_{2}$ at 288 and at $318 \mathrm{~K}$ was linear $(r=0.9985)$. The value of isokinetic temperature evaluated from Exner's plot is $491 \pm 24 \mathrm{~K}$. The linear isokinetic correlation implies that all the alcohols are oxidized by the same mechanism and that changes in rate are governed by the changes in both the enthalpy and entropy of the activation.

The observed hydrogen-ion dependence suggests that the reaction follows two mechanistic pathways, one is acid-independent and the other is acid dependent. Acid catalysis may well be attributed to protonation of $\mathrm{QBC}$ to yield a protonated $\mathrm{Cr}(\mathrm{VI})$ species which is a stronger oxidant and electrophile,

$$
\mathrm{QHOCrO}_{2} \mathrm{Br}+\mathrm{H}^{+} \rightleftharpoons \stackrel{+}{\mathrm{QHOC}}(\mathrm{OH}) \mathrm{OBr} \text {. }
$$

Formation of a protonated $\mathrm{Cr}(\mathrm{VI})$ species has earlier been postulated in the reactions of structurally similar $\mathrm{PCC}^{11}$ and $\mathrm{PFC}^{12}$.

\subsection{Solvent effect}

The rate constants of the oxidation, $k_{2}$, in eighteen solvents $\left(\mathrm{CS}_{2}\right.$ was not considered, as the complete range of solvent parameters was not available) did not yield any significant correlation in terms of the linear solvation energy relationship (LESR) of Kamlet and Taft. $^{13}$

$$
\log k_{2}=A_{0}+p \pi^{*}+b \beta+a \alpha
$$

The data on the solvent effect were analysed in terms of Swain's ${ }^{14}$ equation of cation- and anion-solvating concept of the solvents also.

$$
\log k_{2}=a A+b B+C .
$$

Here $A$ represents the anion-solvating power of the solvent and $B$ the cation-solvating power. $C$ is the intercept term. $(A+B)$ is postulated to represent the solvent polarity. The rates in different solvents were analysed in terms of (5), separately with $A$ and $B$ and with $(A+B)$. 


$$
\begin{aligned}
& \log k_{2}=0.72( \pm 0.04) A+1.89( \pm 0.03) B-3.95 \text {, } \\
& R^{2}=0.9960 ; s d=0.03 ; n=19 ; \psi=0.05 \text {. } \\
& \log k_{2}=0.45( \pm 0.62) A-2.75 \text {, } \\
& r^{2}=0.0296 ; s d=0.50 ; n=19 ; \psi=0.93 \\
& \log k_{2}=1 \cdot 84( \pm 0 \cdot 13) B-3 \cdot 81 \text {, } \\
& r^{2}=0.9211 ; s d=0 \cdot 14 ; n=19 ; \psi=0.21, \\
& \log k_{2}=1 \cdot 50 \pm 0 \cdot 15(A+B)-3 \cdot 88 \text {, } \\
& r^{2}=0.8520 ; s d=0 \cdot 20 ; n=19 ; \psi=0.29 \text {. }
\end{aligned}
$$

Here $n$ is the number of data points and $\psi$ is Exner's statistical parameter. ${ }^{15}$

The rates of oxidation of ethanol in different solvents showed an excellent correlation in Swain's equation (cf. (6)) with the cation-solvating power playing the major role. In fact, the cation-solvation alone accounts for $\approx 92 \%$ of the data. Correlation with the anion-solvating power was very poor. Solvent polarity, represented by $(A+B)$, also accounted for $\approx 85 \%$ of the data.

\subsection{Correlation analysis of reactivity}

The rates of oxidation of the alcohols failed to yield any significant correlation separately with Taft's ${ }^{16} \sigma^{*}$ and $E_{s}$ values.

$$
\begin{aligned}
\log k_{2} & =-1.96( \pm 0 \cdot 33) \Sigma \sigma^{*}-0.61 \\
r^{2} & =0.8329 ; s d=0.49 ; \psi=0.31 ; n=9 . \\
\log k_{2} & =-1 \cdot 07( \pm 0 \cdot 32) \Sigma E_{s}-0.76 \\
r^{2} & =0.6186 ; s d=0.62 ; \psi=0.49 ; n=9 .
\end{aligned}
$$

The rates were, therefore, correlated in terms of Pavelich-Taft's ${ }^{17}$ dual substituentparameter (DSP),

$$
\log k_{2}=\rho^{*} \sigma^{*}+\delta E_{s}+\log k_{0}
$$

The values of substituent constants were obtained from the compilation by Wiberg. ${ }^{16}$ The correlations are excellent; the reaction constants being negative (table 4). There is no significant collinearity $\left(r^{2}=0.2322\right)$ between $\sigma^{*}$ and $E_{s}$ values of the nine substituents.

The negative polar reaction constant indicates an electron-deficient carbon centre in the transition state of the rate-determining step. The negative steric reaction constant shows steric acceleration of the reaction. This may be explained on the basis of the high ground state energy of the sterically crowded alcohols. Since crowding is relieved in the product aldehyde as well as in the transition state leading to it, the transition state energies of the crowded and uncrowded alcohols do not differ much and therefore, results in steric acceleration.

Table 4. Temperature dependence of the reaction constant.

\begin{tabular}{lccccc}
\hline Temp./K & $\rho^{*}$ & $\delta$ & $r^{2}$ & $s d$ & $\psi$ \\
\hline 288 & $-1.61 \pm 0.01$ & $-0.71 \pm 0.01$ & 0.9999 & 0.01 & 0.01 \\
298 & $-1.50 \pm 0.02$ & $-0.63 \pm 0.01$ & 0.9989 & 0.01 & 0.03 \\
308 & $-1.41 \pm 0.01$ & $-0.54 \pm 0.02$ & 0.9998 & 0.01 & 0.01 \\
318 & $-1.33 \pm 0.01$ & $-0.48 \pm 0.01$ & 0.9999 & 0.01 & 0.01 \\
\hline
\end{tabular}




\section{Mechanism}

Presence of a substantial primary kinetic isotope effect confirms the cleavage of an $\alpha-C-$ $\mathrm{H}$ bond in the rate-determining step. The large negative value of the polar reaction constant together with substantial deuterium isotope effect indicates that the transition state approaches a carbocation in its character. Hence the transfer of hydride-ion from alcohol to the oxidant is suggested. The hydride-transfer mechanism is also supported by the major role played by the cation-solvating power of the solvents.

The hydride ion transfer may take place either by a cyclic process via an ester intermediate or by an acyclic one-step bimolecular process. This postulation is supported by an analysis of the temperature dependence of kinetic isotope effect. Kwart and Nickle ${ }^{18}$ have shown that a study of the dependence of the kinetic isotope effect on temperature can be gainfully employed to resolve this problem. The data for protio- and deuterio-ethanols, fitted to the familiar expression

$$
k_{\mathrm{H}} / k_{\mathrm{D}}=A_{\mathrm{H}} / A_{\mathrm{D}} \exp \left(E_{a} / R T\right)^{19-20},
$$

show a direct correspondence with the properties of a symmetrical transition state in which the activation energy difference $\left(\Delta E_{a}\right)$ for $k_{\mathrm{H}} / k_{\mathrm{D}}$ is equal to the zero-point energy difference for the respective $\mathrm{C}-\mathrm{H}$ and $\mathrm{C}-\mathrm{D}$ bonds $(\approx 4.5 \mathrm{~kJ} / \mathrm{mol})$, and the frequency factors and the entropies of activation of the respective reactions are nearly equal. Similar phenomena have also been observed earlier in the oxidation of diols by $\mathrm{BPCC}^{21}$ and that of alcohols by $\mathrm{PFC}^{4}$. Bordwell ${ }^{22}$ has documented very cogent evidence against the occurrence of concerted one-step biomolecular processes by hydrogen transfer and it is evident that in the present studies also, hydrogen transfer does not occur by an acyclic biomolecular process. It is well-established that intrinsically concerted sigmatropic reactions, characterized by transfer of hydrogen in a cyclic transition state, are the only truly symmetrical processes involving linear hydrogen transfer. ${ }^{23}$ Littler $^{24}$ has also shown that a cyclic hydride transfer, in the oxidation of alcohols by $\mathrm{Cr}(\mathrm{VI})$, involves six electrons and, being a Huckel-type system, is an allowed process. Thus the overall mechanism is proposed to involve the formation of a chromate ester in a fast preequilibrium step and then a disproportionation of the ester in a subsequent slow step via a cyclic concerted symmetrical transition state leading to the product (scheme 1). The

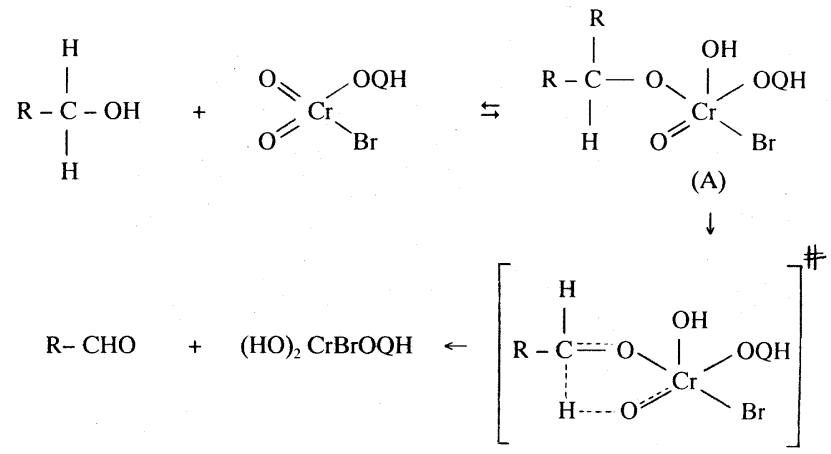

Scheme 1. Acid-independent path. 


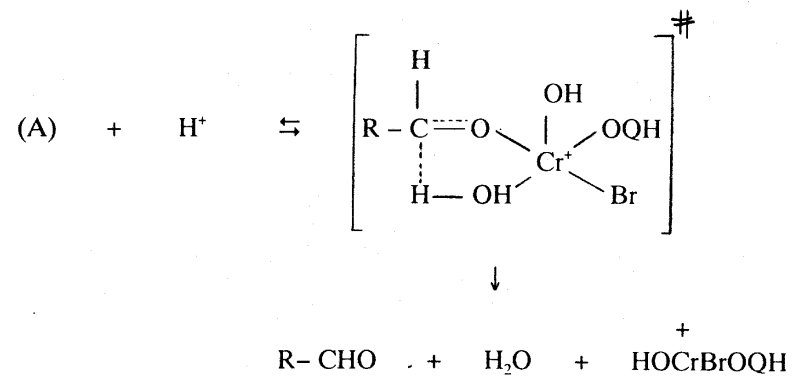

Scheme 2. Acid-dependent path.

observed hydrogen-ion dependence can be explained by assuming a rapid reversible protonation of the chromate ester (A) with the protonated ester decomposing at a rate faster than that of (A) (scheme 2).

It is of interest to compare here the modes of oxidation of alcohols $\mathrm{BPCC}^{2}, \mathrm{QFC}^{3}$ and QBC. Oxidation by QFC exhibited Michaelis-Menten type of kinetics with respect to alcohols, and oxidation by both BPCC and QBC presented similar kinetic pictures. The rate laws, hydrogen-ion dependence and kinetic isotope effect are similar in both the cases. In the oxidation of alcohols by halochromates, excellent correlations were obtained in terms of Swain's equation. Though the data are not conclusive, it seems that the mode of oxidation depends upon the nature of the halogen present in the $\mathrm{Cr}(\mathrm{VI})$ species and not on the nature of the base.

\section{Acknowledgements}

Thanks are due to the University Grants Commission, New Delhi for financial support.

\section{References}

1. Pandurangan A, Murugesan V and Palanichamy M 1995 J. Indian Chem. Soc. 72479

2. Rathore S, Sharma P K and Banerji K K 1995 Indian J. Chem. B34 702

3. Choudhary K, Sharma P K and Banerji K K 1999 Int. J. Chem. Kinet. 31469

4. Banerji K K 1988 J. Chem. Soc., Perkin Trans. 2547

5. (a) Mathur D, Sharma P K and Banerji K K 1993 J. Chem. Soc., Perkin Trans. 2 205; (b) Grover A, Varshney S and Banerji K K 1996 Indian J. Chem. $\mathbf{A 3 5} 206$

6. Kalpan L 1958 J. Am. Chem. Soc. 802639

7. Perrin D D, Armarego W L and Perrin D R 1966 Purification of organic compounds (Oxford: Pergamon)

8. Khanchandani R, Sharma P K and Banerji K K 1995 J. Chem. Res. (S), 242 (M) 1663

9. Murugesan V and Pandurangan A 1995 React. Kinet. Catal. Lett. 54173

10. Exner O 1964 Collect. Czech. Chem. Commun. 291094

11. Bhattacharjee M N, Choudhary M K and Purukayastha S 1987 Tetrahedron 435389

12. Brown H C, Rao C G and Kulkarni S U 1979 J. Org. Chem. 442809

13. Kamlet M J, Abboud J L M, Abraham M H and Taft R W 1983 J. Org. Chem. 48 2877, and references cited therein

14. Swain C G, Swain M S, Powel A L and Alunni S 1983 J. Am. Chem. Soc. 105502

15. Exner O 1966 Collect. Czech. Chem. Commun. 313222

16. Wiberg K B 1963 Physical organic chemistry (New York: Wiley) p. 416

17. Pavelich W A and Taft R W 1957 J. Am. Chem. Soc. 794835 
18. Kwart H and Nickel J H 1973 J. Am. Chem. Soc. 953394

19. Kwart H and Latimer M C 1971 J. Am. Chem. Soc. 933770

20. Kwart H and Slutsky J 1972 J. Chem. Soc., Chem. Commun. 1182

21. Loonker K, Sharma P K and Banerji K K 1997 J. Chem. Res. (S) 242 (M) 1663

22. Bordwell F G 1974 Acc. Chem. Res. 5374

23. Woodward R W and Hoffmann R 1969 Angew. Chem., Int. Ed. Eng. 8781

24. Litller J S 1971 Tetrahedron 2781 